Take the online multiple choice questions associated with this article (see page 765)

S tress testing is a cornerstone in the evaluation of patients with coronary artery disease and its results are always integrated into any clinical decisions. In contrast, valvular heart disease is usually considered static and its management relies upon resting evaluation only. However, most valve diseases have a dynamic component. Changes in loading conditions and contractility during a patient's life may lead to alterations in the severity of lesions, good or poor ventricular contractile reserve, altered volume-dependent compliance of heart chambers, and ventricular arterial coupling. Thus, there may be a need for stress testing and imaging in this setting. Exercise testing in particular can induce symptoms, reveal the dynamics of the valve and the ventricle, and evaluate the changes in forward output, retrograde flow and pulmonary pressures. The current primary role of stress testing in valve disease is to provide an objective assessment of functional disability which is of the utmost importance in patients who often adapt and reduce their physical activity, thus masking their symptoms. The Euro Heart Survey on valvular disease recently showed that stress testing is underused in Europe, or used for inappropriate purposes. ${ }^{1}$

The following questions will be addressed for each of the left valvular heart diseases:

- Who needs stress testing?

- How to test: which stress modality should be used?

- Which parameters should be measured and followed-in other words, should the valve, the ventricle, or both, be tested?

- How can the results help in the patient's management?

\title{
AORTIC STENOSIS
}

Asymptomatic severe aortic stenosis

Who needs stress testing?

Valve replacement is required in the presence of symptoms and severe aortic stenosis. In such patients, stress testing is contraindicated. In contrast, exercise testing is recommended in asymptomatic patients with severe aortic stenosis. Exercise is strongly advocated in the European guidelines ${ }^{2}$ and is a grade IIb recommendation in the American College of Cardiology/American Heart Association (ACC/AHA) 2006 guidelines. ${ }^{3}$ Stress testing has been shown to be low risk when it is performed in asymptomatic patients under strict surveillance. Exercise testing in adults with aortic stenosis has poor diagnostic accuracy for evaluation of concurrent coronary artery disease.

\section{How to test?}

A symptom-limited exercise test is more physiologic than a dobutamine test and may be performed safely. Treadmill or upright bicycle ergometry are the most frequent tests and the choice is based on individual experience. Exercise testing should be repeated every 6 months for severe aortic stenosis and every year for moderate aortic stenosis. Supine or semi-supine bicycle exercise could be preferable, because of a reduced risk of haemodynamic collapse in this position. Symptom-limited graded bicycle exercise in a semi-supine position on a tilting table allows continuous twodimensional and Doppler echocardiographic examination. Dobutamine stress echocardiography may

See end of article for authors' affiliations

Correspondence to: Professor Luc Piérard, Hôpital Universitaire Sart Tilman, Service de Cardiologie, $\mathrm{CHU}$ Sart Tilman, Liege, Belgique; lpierard@chu.ulg.ac.be be used to assess valve compliance by plotting effective orifice area against flow at each stage of the dobutamine test. ${ }^{4}$

Testing should be performed in an appropriately equipped laboratory in the presence of a physician in order that potential complications could be treated effectively.

Which parameters?

Total exercise time, maximum workload, peak heart rate and blood pressure and the reason for stopping the test are recorded. 
Table 1 Criteria of an abnormal exercise test in patients with asymptomatic aortic stenosis

- Symptoms during exercise: dyspnoea, angina, syncope or near syncope

- Fall in blood pressure or $<20 \mathrm{~mm} \mathrm{Hg}$ rise in systolic blood pressure during exercise

$-<80 \%$ of normal level of exercise tolerance

$>2 \mathrm{~mm}$ ST segment depression during exercise (horizontal or downsloping, in comparison to baseline, not attributable to other causes)

- Ventricular arrhythmias

The criteria of an abnormal exercise test provided in the European recommendations are listed in table $1 .{ }^{1}$ It is essential to record the development of symptoms carefully, such as objective dyspnoea, angina, dizziness or near-syncope.

When Doppler echocardiography is obtained during exercise, aortic velocity-time integral can be regularly recorded from the same window to assess changes in mean pressure gradient. The right parasternal window is usually not easily sought in the left lateral cycling position.

\section{Management}

No randomised trial has been conducted in patients with asymptomatic severe aortic stenosis. The risk of sudden death is low and is usually considered to be lower than the risk of operation. However, the mortality is rather high early after the onset of symptoms or if the patient is on a surgical waiting list. In some patients, symptoms are not identified, especially in elderly subjects who are rather inactive. On the other hand, dyspnoea and chest pain may be non-specific.

Exercise testing can identify a limited exercise capacity and reveal symptoms in many (usually one-third) apparently asymptomatic patients. The 1 year prognosis of patients with a normal exercise test is excellent. ${ }^{5}$ In contrast, a positive exercise test predicts the onset of a cardiac event in a sizeable proportion of patients. ${ }^{5-7}$ The development of symptoms during exercise seems to be more predictive than the other criteria of positivity, but this needs to be confirmed.

$A \geqslant 18 \mathrm{~mm} \mathrm{Hg}$ increase in mean transaortic pressure gradient predicts a higher risk of cardiac events and has been shown to provide incremental prognostic information over clinical, resting Doppler echocardiographic and exercise testing. ${ }^{8}$

Exertional symptoms are better predicted by valve compliance during dobutamine stress than by resting Doppler parameters, but the clinical significance of valve compliance requires further studies. ${ }^{4}$

Despite the recommendations to perform exercise testing in patients with asymptomatic severe aortic stenosis and the valuable information obtainable by the test, the recent Euro Heart Survey on valvular heart disease revealed that an exercise test was performed in only $5.7 \%$ of patients in this setting. ${ }^{1}$

\section{Low flow/low gradient aortic stenosis Who needs stress testing?}

Patients with low gradient aortic stenosis and left ventricular dysfunction may have a reduced aortic valve area because of inadequate forward stroke volume due to cardiomyopathyrelative, non-severe aortic stenosis-or may have fixed severe aortic stenosis with afterload mismatch. Stress testing is useful to distinguish patients with fixed aortic stenosis from those with relative aortic stenosis.

\section{How to test?}

In this setting, a dobutamine challenge is recommended to determine the absence or the presence and extent of contractile reserve $^{39}$ (class IIa in the ACC/AHA practice guidelines). A moderate dose of dobutamine should be used in order to obtain the maximal inotropic effect without chronotropic response. Dobutamine is usually started at $5 \mu \mathrm{g} / \mathrm{kg} / \mathrm{min}$ and should be increased to no more than $20 \mu \mathrm{g} / \mathrm{kg} / \mathrm{min}$. The duration of each stage and the mode of dose increment at each step are not standardised; they may vary from 3-5 min and from $2.5-5 \mu \mathrm{g} /$ $\mathrm{kg} / \mathrm{min}$, respectively. Blood pressure and 12 lead ECG need to be carefully monitored, and crash cart and personnel trained in cardiac resuscitation must be present. Criteria for terminating the infusion are the maximal dose, an increase in heart rate $\geqslant 10-20$ beats/min, or an obvious inotropic response.

Dobutamine infusion can be coupled with Doppler echocardiography or with cardiac catheterisation. ${ }^{10}{ }^{11}$ Whatever the method used, the parameters must be carefully and accurately recorded. With Doppler echocardiography, left ventricular outflow tract and aortic velocity-time integrals should be measured for a minimum of three beats in sinus rhythm and up to 10 consecutive beats in atrial fibrillation. In the catheterisation laboratory, simultaneous measurement of left ventricular and aortic pressure is mandatory. Cardiac output should preferably be measured by the Fick method which is more accurate in the presence of a low output. ${ }^{12}$

\section{Which parameters?}

According to the ACC/AHA guidelines, low gradient aortic stenosis is defined by a mean gradient $<30 \mathrm{~mm} \mathrm{Hg}$ and a calculated aortic valve area $<1 \mathrm{~cm}^{2}{ }^{3}$ However, most published studies have included patients with a mean gradient $<40 \mathrm{~mm} \mathrm{Hg}$.

The criteria for dobutamine responsiveness differ among studies. The multicentre French study selected $a \geqslant 20 \%$ increase in forward stroke volume as the cut-off value of contractile reserve; this equates to $a \geqslant 20 \%$ increase in left ventricular outflow tract velocity time integral, as the outflow tract diameter was assumed to be constant. ${ }^{10}$

Other studies have also considered changes in peak aortic velocity, mean pressure gradient, aortic valve area and even wall motion score. Fixed aortic stenosis with contractile reserve is characterised by significant increases in peak velocity and mean gradient without change in valve area (fig 1). Relative aortic stenosis with contractile reserve leads to a significant increase in calculated aortic valve area without change in peak velocity or mean gradient. ${ }^{13}$

\section{Management}

In patients with low gradient aortic stenosis, the operative risk is well stratified by dobutamine stress haemodynamics. Patients with left ventricular contractile reserve and fixed severe aortic stenosis have an acceptable operative risk. ${ }^{10}$ Valve replacement is recommended in most of these patients and usually leads to improved functional status and survival. The precise cut-off values for defining contractile reserve remain uncertain. 


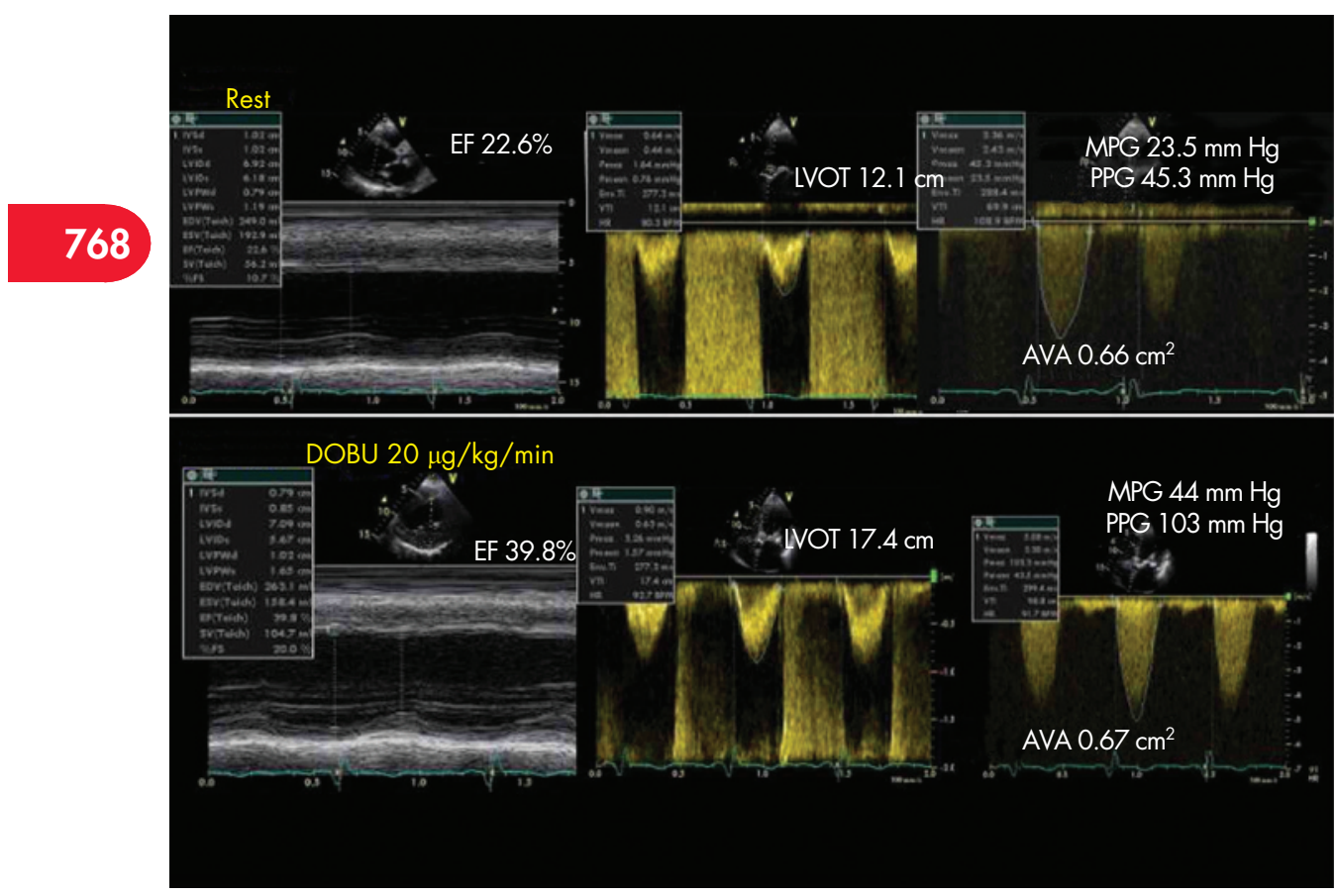

Figure 1 Two dimensional

echocardiography and Doppler findings in a patient with aortic stenosis and severe left ventricular dysfunction. Top: Rest recordings suggest severe aortic stenosis with low pressure gradient. Bottom: Dobutamine stress echo recordings showing that depressed left ventricular function is related to severe aortic stenosis. In this patient, dobutamine at a dosage of $20 \mu \mathrm{g} / \mathrm{kg} /$ min induced a $44 \%(\geqslant 20 \%)$ increase in stroke volume and a significant increase in transvalvular pressure gradient. The calculated aortic valve area is unchanged. AVA, aortic valve area; $E F$, ejection fraction; LVOT, left ventricular outflow tract time velocity integral; MPG, mean transaortic pressure gradient; PPG, peak transaortic pressure gradient.

In the absence of contractile reserve, the operative mortality is high but the outcome is very poor with medical treatment. Therefore, these patients should not necessarily be denied valve surgery. Operative risk appears to be higher when baseline mean pressure gradient is $\leqslant 20 \mathrm{~mm} \mathrm{Hg}$ or associated coronary artery disease is present. The absence of contractile reserve does not predict the absence of left ventricular ejection fraction recovery in patients surviving to aortic valvular replacement. ${ }^{14}$

Patients with relative or pseudo aortic stenosis should be treated medically and not submitted to valve replacement surgery.

\section{AORTIC REGURGITATION Who needs stress testing?}

Asymptomatic patients with severe aortic regurgitation and left ventricular dilation (end-systolic diameter $>50-55 \mathrm{~mm}$ ) may develop irreversible left ventricular damage while awaiting a decision regarding the need for surgery. Stress testing could be useful for the early detection of latent systolic failure (fig 2). When systolic left ventricular dysfunction is obvious, dynamic testing of the left ventricle provides prognostic information. ${ }^{15}$ The test may also be helpful when patients with mild to moderate aortic regurgitation present objective functional limitation. Although the role of exercise stress testing in aortic regurgitation has not yet been demonstrated, it is considered reasonable (class IIA) in the US guidelines for assessment of functional capacity and symptomatic response in patients with a history of equivocal symptoms (level of evidence: B) or before participation in athletic activities (level of evidence: C). ${ }^{3}$

\section{How to test?}

Both exercise and dobutamine stress tests can be performed. Exercise testing provides additional information on exercise performance.

\section{Which parameters?}

Left ventricular end-diastolic and end-systolic volumes and ejection fraction should be assessed at rest and during the test. Annular systolic velocities and indices of longitudinal function could be measured using tissue Doppler imaging ${ }^{16}$ (fig 2).

\section{Management}

Asymptomatic patients with a moderately enlarged left ventricle secondary to severe aortic regurgitation and evidence of latent left ventricular dysfunction (failure to increase left ventricular ejection fraction during test) should be considered for surgery.

\section{MITRAL STENOSIS \\ Who needs stress testing?}

The asymptomatic patient with documented mild mitral stenosis (mean gradient $<5 \mathrm{~mm} \mathrm{Hg}$, valve area $>1.5 \mathrm{~cm}^{2}$ ) should be followed-up on an annual basis, but does not require further evaluation on the initial work-up. When mitral stenosis is significant (valve area $\leqslant 1.5 \mathrm{~cm}^{2}$ ), a haemodynamic stress test should be performed, especially in sedentary patients. This could also be helpful in patients with apparently mild mitral stenosis but who describe limiting symptoms such as dyspnoea. The data regarding the use of stress testing in mitral stenosis are rather limited.

\section{How to test?}

Doppler echocardiography should be used. Dynamic exercise is the most physiologic test and can be done by upright treadmill or supine bicycle. Dobutamine stress testing may also be performed. ${ }^{17}$ The patient should be tested without withdrawal of his or her medical treatment, including digoxin and $\beta$-blocker. 


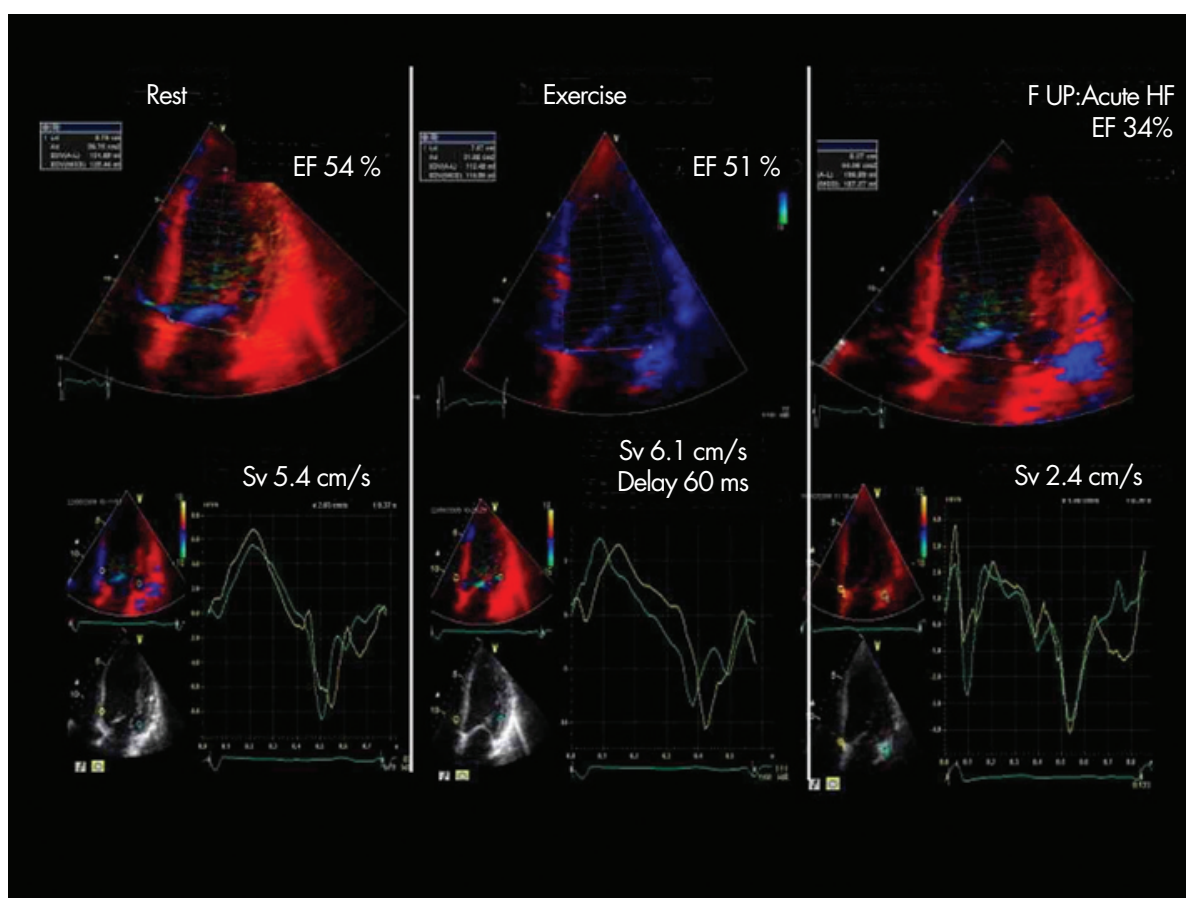

Figure 2 Echocardiographic

assessment of an asymptomatic patient with severe aortic regurgitation. At rest, left ventricular ejection fraction (EF) is normal as well as the peak systolic velocity (Sv) obtained at the level of the septal annulus by tissue Doppler imaging. During exercise, the EF slightly decreases and the increase in Sv is low

\section{Which parameters?}

Exercise tolerance must be evaluated. Doppler recordings of transmitral and tricuspid velocities are obtained allowing measurement of the transmitral gradient and estimation of pulmonary artery systolic pressure during stress (fig 3).

\section{Management}

If mitral valve morphology is suitable for percutaneous balloon valvotomy, patients who deny symptoms but have objective significant limitation of exercise tolerance may be considered for percutaneous valvotomy. This procedure can also be proposed in patients with a valve area $>1.5 \mathrm{~cm}^{2}$ who, during exercise, exhibit a transmitral mean gradient $>15 \mathrm{~mm} \mathrm{Hg}$, pulmonary artery wedge pressure $\geqslant 25 \mathrm{~mm} \mathrm{Hg}$, or pulmonary artery systolic pressure $>60 \mathrm{~mm} \mathrm{Hg}$ (class IIb, level of evidence: $\mathrm{C}$, according to the ACC/AHA guidelines). ${ }^{3}$ When a dobutamine test is performed, the evolution of pulmonary pressure is not helpful. A mean transmitral gradient $>18 \mathrm{~mm} \mathrm{Hg}$ during the test has been shown to be the best cut-off value to predict clinical deterioration or the need to operate. ${ }^{18}$

\section{MITRAL REGURGITATION \\ Organic mitral regurgitation \\ Who needs stress testing?}

Asymptomatic patients with severe (effective regurgitant orifice $(\mathrm{ERO})$ area $\geqslant 40 \mathrm{~mm}^{2}$ ) organic mitral regurgitation are at
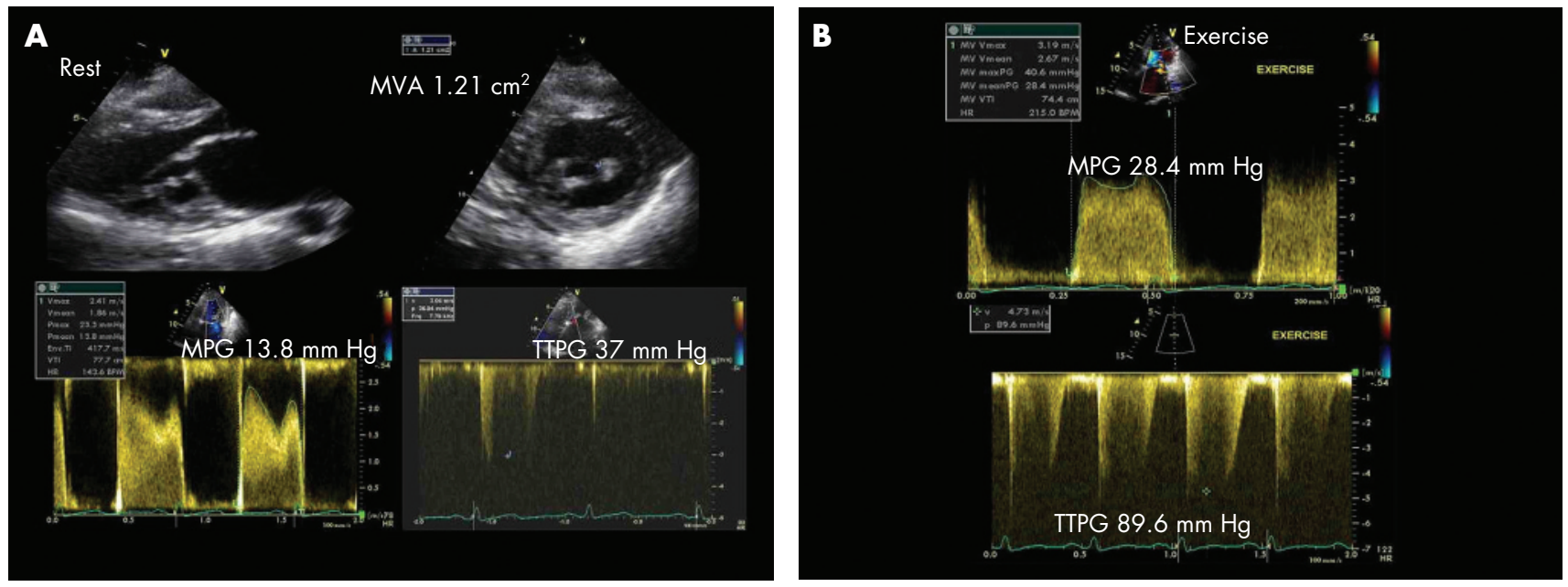

Figure 3 Echocardiographic assessment of an asymptomatic patient with mitral stenosis. (A) Rest recordings suggest significant mitral stenosis.

(B) Exercise recordings show a significant increase in transmitral mean pressure gradient and in transtricuspid pressure gradient. MPG, mean pressure gradient of the transmitral Doppler flow; MVA, mitral valve area; TTPG, transtricuspid pressure gradient. 
increased risk of cardiac events. ${ }^{19}$ Some of them might develop significant left ventricular dysfunction after mitral valve repair despite the presence of a normal preoperative resting left ventricular ejection fraction. Stress testing may be useful for unmasking latent left ventricular contractile dysfunction and predicting postoperative ejection fraction..$^{20}$ The published data are limited. Exercise Doppler echocardiography is reasonable in asymptomatic patients with severe mitral regurgitation to assess exercise tolerance and the effects of exercise on pulmonary artery pressure and mitral regurgitation severity (class IIa, level of evidence: C). ${ }^{3}$

\section{How to test?}

Both post- and per-exercise (treadmill, bicycle) imaging can be used. The value of dobutamine stress testing has not yet been investigated, but this mode of testing is not ideal as dobutamine would further reduce afterload.

\section{Which parameters?}

Exertional symptoms must be recorded. Left ventricular enddiastolic volume, end-systolic volume, ejection fraction and transtricuspid pressure gradient (non-invasive estimation of pulmonary artery systolic pressure) should be assessed at rest and during testing.

\section{Management}

Not all patients with asymptomatic severe organic mitral regurgitation are good candidates for early surgery because of advanced age, comorbidities, or unrepairable valves. In these patients, a more conventional strategy of watchful waiting remains a reasonable option in the absence of latent left ventricular dysfunction-exercise-induced increases in left ventricular ejection fraction by $4 \%$ or left ventricular endsystolic volume index $\geqslant 25 \mathrm{~cm}^{3} / \mathrm{m}^{2}$ at exercise. ${ }^{2021}$ Elective surgery might also be proposed in patients with a high likelihood of valve repair and exercise-induced systolic pulmonary arterial pressure $>60 \mathrm{~mm} \mathrm{Hg}$ (class IIa, level of evidence: C).

\section{Ischaemic mitral regurgitation}

Ischaemic mitral regurgitation is a left ventricular disease and develops in the presence of a structurally normal mitral valve. It results from apical and outward displacement of the papillary muscles tethering the mitral leaflets and from a decreased left ventricular generated force to close them. The clinical importance of ischaemic mitral regurgitation is underestimated, at least partly because physical examination is insensitive. When present, a systolic murmur is usually soft even when mitral regurgitation becomes severe. Functional, ischaemic mitral regurgitation varies dynamically in accordance with changes in loading conditions, annular size and the balance of tethering versus closing forces. ${ }^{22}$

\section{Who needs stress testing?}

Most patients presenting with ischaemic mitral regurgitation have a prior history of myocardial infarction, left ventricular dilation and reduced left ventricular ejection fraction. The dynamic component of ischaemic mitral regurgitation might be tested:
- in patients with left ventricular systolic dysfunction who present exertional dyspnoea out of proportion to the severity of resting dysfunction or mitral regurgitation

- in patients in whom acute pulmonary oedema occurs without an obvious cause

- for stratifying the risk of mortality and heart failure decompensation in the individual patient

- before surgical revascularisation in patients with moderate mitral regurgitation.

However, stress testing is not yet recommended in this setting by the ACC/AHA 2006 guidelines.

\section{How to test?}

Candidates for combined coronary artery bypass grafting and mitral valve repair should not be selected by transoesophageal echocardiography in the operating room because general anaesthesia results in reduced loading conditions and tethering, and thus largely underestimates mitral regurgitation. Similarly, dobutamine stress reduces preload, afterload and mitral regurgitation, and is therefore not useful. Dynamic exercise testing should be used, ideally, on a dedicated exercise table.

\section{Which parameters?}

Numerous Doppler echocardiographic variables could be recorded both at baseline and during exercise and stored for off-line detailed analysis. Left ventricular parameters include end-diastolic and end-systolic volumes and ejection fraction, regional wall thickening, and colour-coded Doppler tissue imaging to reconstitute and analyse off-line pulsed-wave Doppler velocity profiles. Mitral valve deformation can be quantitated by measuring the systolic tenting area (the area enclosed between the annular plane and the mitral leaflets in the parasternal long-axis view), displacement of mitral coaptation toward the left ventricular apex (the distance between leaflet coaptation and the mitral annulus plane in the apical four-chamber view) and the distance between the posterior papillary muscle and the intervalvular fibrosa in the apical long-axis view. Mitral regurgitation should be quantitated at rest and during exercise (fig 4). The regurgitant jet area is not reproducible and should preferably not be used. ${ }^{23}{ }^{24}$ The PISA (proximal isovelocity surface area) method is reproducible and reliable if the flow-convergence region is appropriate. The Doppler method is an alternative in patients with a suboptimal flow-convergence definition. Regurgitant volumes calculated by the Doppler method are usually slightly larger than those obtained with the PISA method. ${ }^{24}$ The results of the two methods may therefore be averaged. The ERO area appears to be the most robust parameter for quantifying mitral regurgitation at rest and during exercise.

Systolic pulmonary artery pressure and left ventricular $\mathrm{dP} / \mathrm{dt}$ can be estimated from the systolic transtricuspid pressure gradient and the steepest increasing segment of the continuous wave Doppler mitral regurgitant jet, respectively.

An ERO area $\geqslant 20 \mathrm{~mm}^{2}$ is considered severe and is associated with excess mortality. ${ }^{25}$ The degree of mitral regurgitation at rest is unrelated to exercise-induced changes in ERO, ${ }^{26}$ which are related to those in mitral valve deformation (a marker of tethering force) and to those in left ventricular dyssynergy (an indicator of closing force). ${ }^{27} 28$ 


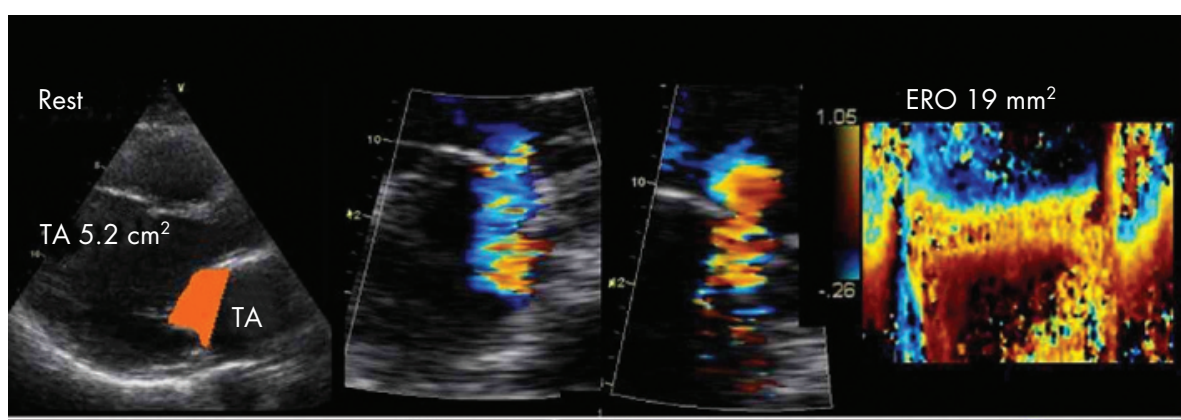

Figure 4 End-systolic stop frame images and proximal flow-convergence region at rest and during exercise in a patient with chronic posterior myocardial infarction and functional mitral regurgitation. Top: At rest, the systolic tenting area (TA) is enlarged and mitral regurgitation is moderate. The colour M-mode reveals a characteristic pattern of the proximal flow convergence zone with early and late systolic peaks and a mid-systolic decrease in the PISA (proximal

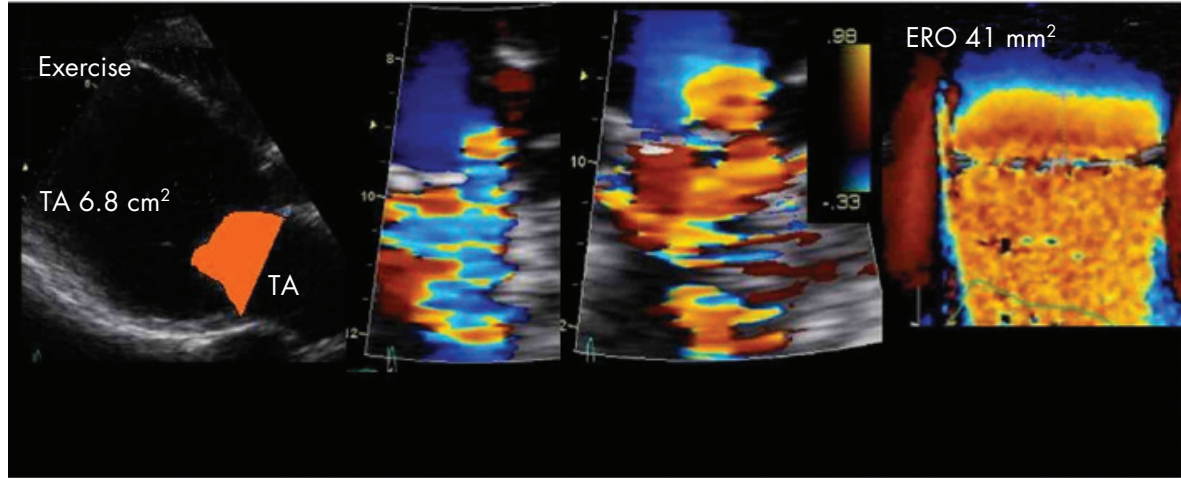
isovelocity surface area) radius. Bottom: During exercise, the severity of mitral regurgitation increases greatly. The proximal flow convergence zone becomes larger and the radius is nearly constant throughout systole. ERO, effective regurgitant orifice area.

Dynamic functional mitral regurgitation usually develops in the absence of transient induced ischaemia.

An exercise-induced increase in mitral regurgitation is larger in patients who stop their exercise test because of dyspnoea as compared to those who stop because of fatigue. ${ }^{29}$ Parallel increases in ERO and pulmonary pressures characterise patients

\section{Stress testing in valve disease: key points}

- Most valvular heart diseases have a dynamic component

- Exercise testing, exercise echocardiography and dobutamine stress echocardiography are the most frequent stress modalities

- Exercise testing is recommended in asymptomatic severe aortic stenosis. A normal exercise test predicts good short term (1 year) outcome

- In symptomatic patients with low gradient aortic stenosis, dobutamine stress echocardiography should be performed to distinguish relative from fixed aortic stenosis. Patients with fixed aortic stenosis, but without contractile reserve by dobutamine stress testing, have a high operative mortality compared with patients with contractile reserve

- In patients with mitral stenosis, the severity of exerciseinduced increases in mean pressure gradient and estimated pulmonary pressures can help in decision making

- Post-exercise echocardiography helps to identify patients operated for severe organic mitral regurgitation who are at risk of postoperative left ventricular dysfunction. They are characterised by a larger left ventricular end-systolic volume index and a low contractile reserve

- Ischaemic mitral regurgitation is characteristically dynamic. Exercise-induced changes in effective regurgitant orifice (ERO) area are not predictable by any measurement made at rest

- A large increase in ERO area is associated with a high risk of mortality and of decompensated heart failure with systolic heart failure who may develop acute pulmonary oedema. ${ }^{30}$

An increase in ERO $\geqslant 13 \mathrm{~mm}^{2}$ during exercise is associated with both mortality and hospital admission for worsening heart failure. ${ }^{31}{ }^{32}$ Patients who exhibit a sizeable decrease in ERO because of recruitable contraction of the basal left ventricular segments have a good long-term prognosis.

\section{Management}

Patients with severe functional mitral regurgitation at rest and dynamic mitral regurgitation should receive optimal medical treatment, including an angiotensin-converting enzyme (ACE) inhibitor and a $\beta$-blocker that can contribute to reverse left ventricular remodelling and reduction in mitral regurgitation. When the QRS width is enlarged (>120 ms) and significant left ventricular dyssynchrony is demonstrated, patients will benefit from cardiac resynchronisation therapy which acutely decreases mitral regurgitation and its dynamic component, and might contribute to long-term reverse remodelling and further reductions in mitral regurgitation. ${ }^{33} 34$

In patients who need surgical revascularisation, the role of mitral valve surgery in the presence of mitral regurgitation remains controversial. Combination treatment involving bypass and mitral ring annuloplasty and specific subvalvular approaches might be proposed if the ERO is $\geqslant 20 \mathrm{~mm}^{2}$ and/or if $a \geqslant 13 \mathrm{~mm}^{2}$ increase in ERO develops with exercise, but this strategy needs to be tested prospectively.

\section{INTERACTIVE MULTIPLE CHOICE QUESTIONS}

This Education in Heart article has an accompanying series of six EBAC accredited multiple choice questions (MCQs).

To access the questions, click on BMJ Learning: Take this module on BMJ Learning from the content box at the top right and bottom left of the online article. For more information please go to: http://heart.bmj.com/misc/education.dtl 
Please note: The MCQs are hosted on BMJ Learning-the best available learning website for medical professionals from the BMJ Group.

If prompted, subscribers must sign into Heart with their journal's username and password. All users must also complete a one-time registration on BMJ Learning and subsequently log

772 in (with a BMJ Learning username and password) on every visit.

\section{Authors' affiliations}

Luc Piérard, Faculté de Médecine, Université de Liège, Chef de Service, Service de Cardiologie, CHU Sart Tilman, Liege, Belgique

P Lancellotti, Responsable de I'Unité de Soins Intensifs Cardiologiques, CHU Sart Tilman, Liege, Belgique

In compliance with EBAC/EACCME guidelines, all authors participating in Education in Heart have disclosed potential conflicts of interest that might cause a bias in the article

\section{REFERENCES}

1 lung B, Baron G, Butchart EG, et al. A prospective survey of patients with valvular heart disease in Europe: the Euro Heart Survey on valvular heart disease. Eur Heart J 2003;24:1231-43.

2 lung B, Gohlke-Bärwolf C, Tornos P, et al. Recommendations on the management of the asymptomatic patient with valvular heart disease. Eur Heart $J$ 2002;23:1253-66.

3 Carabello BA, Chatterjee K, de Leon AC Jr, et al. ACC/AHA 2006 guidelines for the management of patients with valvular heart disease. J Am Coll Cardiol 2006;48:el-148.

4 Das $\mathrm{P}$, Rimington $\mathrm{H}$, Smeeton $\mathrm{N}$, et al. Determinants of symptoms and exercise capacity in aortic stenosis: a comparison of resting haemodynamics and valve compliance during dobutamine stress. Eur Heart 2003;24:1254-62.

5 Das $\mathbf{P}$, Rimington H, Chambers J. Exercise testing to stratify risk in aortic stenosis. Eur Heart J 2005;26:1309-13.

- Important study concerning the role of exercise testing in aortic stenosis.

6 Amato MCM, Moffa PJ, Werner KE, et al. Treatment decision in asymptomatic aortic valve stenosis: role of exercise testing. Heart 2001;86:381-6.

7 Alborino D, Hoffmann JL, Fournet PC, et al. Value of exercise testing to evaluate the indication for surgery in asymptomatic patients with valvular aortic stenosis. $J$ Heart Valve Dis 2002;1 1:204-9.

8 Lancellotti P, Lebois F, Simon M, et al. Prognostic importance of quantitative exercise Doppler echocardiography in asymptomatic valvular aortic stenosis. Circulation 2005;112(9 Suppl):1377-82.

9 Lange RA, Hillis LD. Dobutamine stress echocardiography in patients with lowgradient aortic stenosis. Circulation 2006:113:1718-20.

10 Monin JL, Quere JP, Monchi M, et al. Low-gradient aortic stenosis: operative risk stratification and predictors for long-term outcome: a multicenter study using dobutamine stress hemodynamics. Circulation 2003;108:319-24.

- Largest multicentre study defining the role of dobutamine stress echocardiography in low gradient aortic stenosis.

11 Nishimura RA, Grantham JA, Connolly HM, et al. Low-output, low-gradient aortic stenosis in patients with depressed left ventricular systolic function: the clinical utility of the dobutamine challenge in the catheterization laboratory. Circulation 2002; 106:809-13.

12 Grayburn PA, Eichhorn EJ. Dobutamine challenge for low-gradient aortic stenosis. Circulation 2002;106:763-5.

13 defilippi CR, Willett DL, Brickner ME, et al. Usefulness of dobutamine echocardiography in distinguishing severe from non-severe valvular aortic stenosis in patients with depressed left ventricular function and low transvalvular gradients. Am J Cardiol 1995;75:191-4.
14 Quere J-P, Monin J-L, Levy F, et al. Influence of preoperative left ventricular contractile reserve on postoperative ejection fraction in low-gradient aortic stenosis. Circulation 2006;113:1738-44.

15 Wahi S, Haluska B, Pasquet A, et al. Exercise echocardiography predicts development of left ventricular dysfunction in medically and surgically treated patients with asymptomatic severe aortic regurgitation. Heart 2000;84:606-14.

16 Vinereanu D, lonescu AA, Fraser AG. Assessment of left ventricular long axis contraction can detect early myocardial dysfunction in asymptomatic patients with severe aortic regurgitation. Heart 2001;85:30-6.

17 Cheitlin MD. Stress echocardiography in mitral stenosis: when is it useful? J Am Coll Cardiol 2004;43:402-4

18 Reis G, Motta MS, Barbosa MM, et al. Dobutamine stress echocardiography for noninvasive assessment and risk stratification of patients with rheumatic mitral stenosis. J Am Coll Cardiol 2004;43:393-401.

19 Enriquez-Sarano M, Avierinos JF, Messika-Zeitoun D, et al. Quantitative determinants of the outcome of asymptomatic mitral regurgitation. N Engl J Med 2005;352:875-83

- Important study demonstrating the importance of quantitation of mitral regurgitation.

20 Leung DY, Griffin BP, Stewart WJ, et al. Left ventricular function after valve repair for chronic mitral regurgitation: predictive value of preoperative assessment of contractile reserve by exercise echocardiography. J Am Coll Cardiol contractile reserve by

- Largest single centre study defining the role of post-exercise echocardiography in organic mitral regurgitation.

21 Lee R, Haluska B, Leung DY, et al. Functional and prognostic implications of left ventricular contractile reserve in patients with asymptomatic severe mitral regurgitation. Heart 2005;91:1407-12.

22 Levine RA, Schwammenthal E. Ischemic mitral regurgitation on the threshold of a solution: from paradoxes to unifying concepts. Circulation 2005; 112:745-58.

- Excellent review of ischaemic mitral regurgitation.

23 McCully RB, Enriquez-Sarano M, Tajik AJ, et al. Overestimation of severity of ischemic/functional mitral regurgitation by color Doppler jet area. Am J Cardio 1994;74:790-3.

24 Lebrun F, Lancellotti P, Piérard LA. Quantitation of functional mitral regurgitation during bicycle exercise in patients with heart failure. J Am Coll Cardiol 2001;38:1685-92.

- First study validating quantitation of mitral regurgitation during exercise.

25 Grigioni $\mathbf{F}$, Enriquez-Sarano M, Zehr KJ, et al. Ischemic mitral regurgitation. Long-term outcome and prognostic implications with quantitative Doppler assessment. Circulation 2001;103:1759-64.

26 Lancellotti P, Lebrun F, Piérard LA. Determinants of exercise-induced changes in mitral regurgitation in patients with coronary artery disease and left ventricular dysfunction. J Am Coll Cardiol 2003:42:1921-8.

27 Lancellotti P, Stainier PY, Lebois F, et al. Effects of dynamic changes in left ventricular synchronicity on dynamic mitral regurgitation. Am J Cardiol 2005;96:1304-7

28 Piérard LA, Lancellotti P. Left ventricular dyssynchrony and dynamic functional mitral regurgitation: relationship or association? Eur Heart J 2006:27:638-40.

29 Piérard LA, Lancellotti P. Dyspnea and stress testing. N Engl J Med 2006;354:871-3.

30 Piérard LA, Lancellotti P. The role of ischemic mitral regurgitation in the pathogenesis of acute pulmonary edema. N Engl J Med 2004;351:1627-34.

- Clinical study indicating that acute pulmonary oedema can be produced by dynamic ischaemic mitral regurgitation.

31 Lancellotti $\mathbf{P}$, Troisfontaines $\mathrm{P}$, Toussaint A-C, et al. Prognostic importance of exercise-induced changes in mitral regurgitation in patients with chronic ischemic left ventricular dysfunction. Circulation 2003;108:1713-7.

32 Lancellotti P, Gérard P, Piérard L. Long term outcome of patients with heart failure and dynamic mitral regurgitation. Eur Heart J 2005;26:1528-32.

- Dynamic ischaemic mitral regurgitation predicts both increased mortality and decompensated heart failure.

33 Breithardt OA, Sinha AM, Schwammenthal E, et al. Acute effects of cardiac resynchronization therapy on functional mitral regurgitation in advanced systolic heart failure. J Am Coll Cardiol 2003;41:765-70.

34 Lancellotti P, Mélon P, Sakalihasan N, et al. Effects of cardiac resynchronization therapy on functional mitral regurgitation in heart failure. Am J Cardiol 2004;94:462-5. 